TITLE:

\title{
Use of video system and its effects on abnormal behaviour in captive Japanese macaques (Macaca fuscata)
}

\author{
$\operatorname{AUTHOR}(\mathrm{S}):$
}

Ogura, Tadatoshi

\section{CITATION:}

Ogura, Tadatoshi. Use of video system and its effects on abnormal behaviour in captive Japanese macaques (Macaca fuscata). Applied Animal Behaviour Science 2012, 141(3-4): 173-183

ISSUE DATE:

2012-11

URL:

http://hdl.handle.net/2433/160401

\section{RIGHT:}

C 2012 Elsevier B.V.; この論文は出版社版でありません。引用の際には 出版社版をご確認ご利用ください。; This is not the published version. Please cite only the published version. 
1

2

3 Use of video system and its effects on abnormal behaviour in captive Japanese macaques

(Macaca fuscata)

5

6

7

Tadatoshi Ogura

8

9

Wildlife Research Center, Kyoto University, Sakyo, Kyoto 606-8203, Japan

12 Correspondence to:

13 Tadatoshi OGURA

14 Wildlife Research Center, Kyoto University, 2-24 Tanaka-Sekiden, Sakyo, Kyoto, Kyoto

15 606-8203, Japan

16 Tel: +81-75-771-4393

17 Fax: +81-75-771-4394

18 E-mail: ogura@wrc.kyoto-u.ac.jp 
have few opportunities to exert such abilities in captivity. Video presentation can reproduce multiple features of the complex real visual world. Therefore, video presentation can be expected to act as environmental enrichment for captive primates. The present study evaluated the enriching effects of novelty and content of videos as well as control over videos using newly developed technology including network-shared YouTube videos and an infrared distance sensor. Baseline data were obtained for 10 days without video display and then 10 days with the display

9 showing no videos in four individually housed monkeys and six pair-housed monkeys. The monkeys were then exposed to videos of conspecifics, people, and animation and observed for

1130 days. In some days, the monkeys had control over videos such that videos played only when subjects sat directly in front of the display. Observations were then taken for an additional 10 days with no videos. Behaviors such as time watching videos, abnormal behavior, and time spent in front of display were recorded for 60 min per day using instantaneous sampling with a 30-s interval. Monkeys displayed fewer abnormal behaviors when the video was playing (individually housed: 11.3 sampling points, pair-housed: 7.7 sampling points) than when

17 the video was not playing (individual housed: 22.5 sampling points, pair-housed: 10.8 
1 showed no evidence of habituation to videos. The frequency of watching the videos and

2 abnormal behaviour differed depending on the presented contents of videos $(P<0.001)$. The

3 subjects spent more time in front of the display in the days in which they could control

4 the videos (individually housed: 89.1 sampling points, pair-housed: 17.6 sampling

5 points) than in those in which they had no control (individually housed: 70.1 sampling

6 points, pair-housed: 7.3 sampling points $)(P<0.001)$, suggesting that they were

7 preferentially choosing to watch the videos. These results suggest that video presentation

8 can be a useful technique to decrease abnormal behaviour of captive Japanese macaques. The

9 content and controllability over videos were influential on their behaviour, suggesting that

10 implementation of video enrichment that includes conspecifics videos and controllability over

11 videos can improve animal welfare. Implementation based on an understanding of

12 species-specific characteristics can contribute to effective environmental enrichment.

13 Keywords: Animal welfare; Cognition; Control over environment; Japanese macaques; Video 


\subsection{Video presentation as environmental enrichment for captive nonhuman primates} and Schapiro, 1991). The decision about which environmental enrichment is optimal depends on the species-specific characteristics of the animals (Kreger et al,, 1998). Since primates have highly developed visual cognitive abilities, video presentation is widely used to provide sensory and cognitive enrichment for primates in research facilities (Reinhardt, 2010) and zoos (Clay et al., 2011). Some previous studies investigated the effect of video presentation on the welfare of captive primates. Platt and Novak (1997) showed that rhesus macaques became more active as a result of video-stimulation. The monkeys engaged in behaviours related to exploration of the

11 environment more frequently during video-stimulation. In chimpanzees, television can attract the animals' interest even after 2 years of exposure (Brent and Stone, 1996). Individually housed chimpanzees were more likely to attend to video presentations than socially housed chimpanzees (Bloomsmith and Lambeth, 2000). Even in a zoo setting, video presentation can still be useful. Silverback western lowland gorillas spent much time watching a monitor when it displayed videos (Maloney et al., 2011). These studies indicated that video presentation may be a useful enrichment technique for captive primates. 
1 primates, its ability to be adjusted to species-specific needs remains to be tested. In the present

2 experiment, key components affecting the enriching effect of videos, such as the novelty and contents of videos and the controllability over videos were examined by using newly developed technology.

\subsection{Novelty and contents of videos}

6 Novelty and contents have been studied as key properties of videos that might affect animals' preference in some previous studies. It is well known that animals become habituated to sensory stimuli which are presented repeatedly and dishabituated to new stimuli (e.g., Koba and Izumi, 2008; Murai et al., 2004; Quinn et al., 2002). Captive primates rapidly become habituated to videos that are presented repeatedly (Bloomsmith and Lambeth, 2000; Lee et al., 2011; Ogura and Matsuzawa, 2012; Platt and Novak, 1997). Novelty affects the attractiveness of videos, and therefore might influence their effect as environmental enrichment for primates. In other words, animals might prefer to watch novel videos than familiar ones. The content of visual stimuli has also been found to affect the preference of primates. In cognitive studies, bonnet macaques (Andrews and Rosenblum, 2001; Brannon et al., 2004) and rhesus macaques (Washburn et al., 1997) preferred to watch videos with particular contents over other videos. Despite the consistent results from these cognitive studies, other studies 
1 (Bloomsmith and Lambeth, 2000; Maloney et al., 2011; Platt and Novak, 1997) found that

2 videos which were supplied as visual enrichment did not affect the behaviour, another study

3 (Ogura and Matsuzawa, 2012) found that videos with varying content did differentially affect

4 the behaviour in Japanese macaques. Thus, the effects of the contents of visual stimuli have not been entirely consistent in enrichment compared to cognitive studies.

6 The present study investigated the effects of novelty and content of videos on the welfare of captive Japanese macaques. To supply a large number of novel videos showing various contents, this study tested the use of videos shared on the internet by anonymous people.

\subsection{Controllability over videos} have found that having control over the environment is important for monkeys. In Washburn et al. (1991), rhesus monkeys performed significantly better on computer tasks they selected compared to identical tasks assigned by the experimenter. Hanson et al. (1976) found that rhesus monkeys that had control over a high-intensity noise had significantly lower plasma cortisol concentrations than monkeys exposed to identical amounts of high-intensity noise but without any control. Another phenomenon is contrafreeloading, which experimentally evaluates the value of control as well as reward which can be obtained as a result of control (Inglis et al., 
1 visual reward (Ogura, 2011) as well as a food reward (Menzel, 1991; Reinhardt, 1994) in

2 primates. These studies provided empirical evidence of the value of control over the

3 environment for primates. To make easy for monkeys to understand a contingency between their behaviour and videos, this study utilized a new technique that allowed the monkeys to control videos by altering their location in their cage.

7

\section{Methods}

\subsection{Subjects}

11 of Kyoto University. Two of the subjects were individually housed males, each 11 years of age, two were individually housed females, 10 and 13 years of age, and the other six were housed in

13 three, male-female pairs. The ages of the pair-housed subjects were not known because they were born in the wild, but they were fully matured. The individually housed subjects had been maintained in cages $(80 \mathrm{~cm}$ high $\times 80 \mathrm{~cm}$ wide $\times 60 \mathrm{~cm}$ long) for more than 2 years. Because of

16 limitations of available space, the subjects could not see other monkeys during this study. The

17 pair-housed subjects were maintained in cages $(200 \mathrm{~cm}$ high $\times 137 \mathrm{~cm}$ wide $\times 115 \mathrm{~cm}$ long $)$ that 
1

2

observer for at least 2 weeks before the start of observations. The monkeys were fed monkey pellets and sweet potatoes daily at about 10:00 h. and 17:00 h and had access to water ad libitum. Routine care of the monkeys and experiments were performed in accordance with the Guide for the Care and Use of Laboratory Primates produced by Primate Research Institute, Kyoto University.

\subsection{Apparatus}

The apparatus in this experiment consisted of a laptop computer connected to a colour display $(30.4 \mathrm{~cm} \times 22.8 \mathrm{~cm})$ (model LCD-A15GS, I-O DATA, Ishikawa, Japan), and an infrared distance (IRD) sensor. The computer displayed the different content of videos according to the experimental conditions and turn on and off the videos based on signals from the IRD sensor. The IRD sensor consisted of a sensor module (GP2Y0A21YK0F, Sharp, Osaka, Japan) and a microcomputer (Arduino Duemilanove, Smart Projects, Turin, Italy). The IRD sensor emitted infrared laser light to the centre of the bottom of the cage and calculated the distance between the apparatus and the subject who cut off the infrared laser light by sitting in front of it. The whole apparatus was small enough to be carried on a cart. It was set up $50 \mathrm{~cm}$ in front of the cage only during the experiment and was removed at other times not to preclude animal access or husbandry activities.

\subsection{Videos}


2 the beginning of each experimental session, the YouTube site was searched using the keyword

3 "Japanese macaques", "people", or "animation" and the results were sorted by uploaded date.

4 Thirty of the most recently uploaded video clips were shown in a day. Because the novelty of

5 the videos affects the macaques' interest in the videos (e.g., Ogura and Matsuzawa, 2012), the

6 search was conducted at the beginning of every session to make a unique video set with newly

$7 \quad$ uploaded video clips. Any video clips that had already been used in past session were replaced

8 with other, older video clips that had not been used.

video clip finished, the next one in the video set was played. The first clip in the video set was

11 played again after all other video clips were finished. All video clips were played at least twice in a day. The construction of the video set and the playback of the video clips were automatically controlled by the computer program produced by the author. The sound from the video clips was omitted from the playback.

\subsection{Procedures}

Each subject experienced 60 observation sessions with one session per day. The sessions started between 12:30 h. and 17:00 h. and continued for 60 min. The observation 
1 monitor, monitor with video, and no-monitor 2. In the no-monitor phases, baseline data were

2 collected without the presence of the apparatus for 10 days. After the first no-monitor phase,

3 monkeys were habituated to the physical presence of an apparatus with a blank monitor near

4 their cages ("blank monitor phase") for 10 days. After that, the "monitor with video" phase was

5 started in which video clips were played on the monitor for 30 days. The "monitor with video"

6 phase consisted of two types: controllable and non-controllable. A different kind of video

7 ("Japanese macaques", "people", or "animation") was used for each of the two types, for a total

8 of six conditions of combinations of video content and controllability. In the controllable

9 condition, the videos were shown on the display only when the subject cut the laser light of the

10 IRD sensor by staying at the centre of the front half of the cage (i.e., the "video zone"). When

11 monkeys want to watch something, it is a very natural response to reach toward it. When the subject left this zone, the videos turned off. Therefore, the duration of the video presentation depended on the subject's location in the cage. In the non-controllable condition, the videos were shown on the display continuously irrespective of the monkey's location in the cage. Each condition consisted of five successive days. The order of the conditions was randomized among the subjects. In the final phase, a second no-monitor phase was conducted again for 10 days. During this testing procedure, the behaviour and cage location of each subject was 
1

2 Thus, the number of sampling points per day was 120. There were seven categories of

were collected using an instantaneous sampling method with a 30-s interval (Altmann, 1974). behaviours: abnormal behaviour, feeding, locomotion, self-grooming, social behaviour, watching video, and resting (Table 1). The definitions of abnormal behaviours were based on Mallapur and Choudhury (2003) and Walsh et al. (1982). For the location data, each cage was divided into two zones: the "video zone" defined as the centre of the front half of the cage and the "non-video zone" (the rest of the cage). While the two zones were different in size, video zones were the same for both individual and pair-housing cages. This does not affect the analyses because the duration of time spent in the video zone in the different conditions was compared within the individually housed or pair-housed subjects. The same observation procedure was employed in both individually housed and pair-housed subjects.

\subsection{Statistical analyses}

The number of sampling points (frequency) for which the subjects engaged in each

behavioural category and stayed in the video zone were analyzed using a Generalized Linear

Mixed Model (GLMM) (lmer, lme4 library, the freeware package R, Version 2.14.2; R 
1 Development Core Team, 2012). This method is an extension of the ANOVA technique,

2 incorporating and quantifying multiple sources of variation and also allowing for non-normal

3 distributions for the response variable. Therefore, the GLMM technique can analyze repeated measures data that are not normally distributed. The models were constructed using a Poisson distribution because the numbers of sampling points were non-negative count data (Dobson, 2002). One data point indicated the score of each behaviour in each day. The data could have been analyzed as proportions of the total counts of a day under the Binomial distribution, but the residual plots from each analysis indicated the models using the Poisson distribution were a

9 better fit with the observation data than the models using the Binomial distribution. In analyses of the effect of the video presentation on the frequency of each behaviour, the number of each

11 behaviour in each day was the response variable. The model contained the experimental phase as the fixed factor. To test the effect of habituation to the video presentation, as well as the contents and controllability of videos, the models contained the frequency of subjects' behavior and the frequency at which the subject stayed in the video zone as the response variables. The fixed factors of the models were the observation day, the content, and the controllability of videos. The individual, sex, and pairing (for the pair-housed subjects) were contained in all

17 GLMM analyses as the random factor to control for repeated measures. Therefore, the 
1 including the target fixed factor and the models without it were compared based on the Akaike

2 Information Criterion (AIC; Akaike, 1974; Dobson, 2002). The model with the lower AIC is

3 preferred. The significance of difference of fitted models was tested using a likelihood ratio test.

4 For multiple comparisons of the fixed factors, the models which contained every combination of

$5 \quad$ the fixed factors were also compared.

6

3. Results

8

9

\subsection{The effect of video presentation on behaviour}

The frequency of abnormal behaviour was the lowest during the "monitor with video" phase for both individually housed and pair-housed subjects. Fig. 1 shows the frequency of abnormal behaviour in all four experimental phases. The models that included the experimental phase as the fixed factor showed significantly lower AICs than the models that did not include it (Table 2). For both single and pair-housed subjects, the models that included the "monitor with video" phase at a different level from the other three phases showed the lowest AICs (2773 and 1822) in the multiple comparisons of the experimental phases. This result suggests that the "monitor with video" phase affected the frequency of abnormal behaviour differently than the other three phases for both the individually housed and pair-housed monkeys. 
1

2
Fig. 1 and Table 2 about here

In the analyses of the other behavioural categories, only resting behaviour for the

behaviour in the "monitor with video" phase $(45.1 \pm 1.6$ sampling points $)$ was lower than in the other experimental phases (no-monitor 1: $54.6 \pm 3.0$, blank monitor: $46.3 \pm 2.8$, no-monitor 2 :

$47.1 \pm 2.9$ ). The model that included the experimental phase as a fixed factor (AIC: 1593) showed a significantly lower AIC than the model without it (AIC: 1643$)\left(\chi^{2}=56.01, P<0.001\right)$. The models that included the "monitor with video" phase at a different level from the other three phases showed the lowest AIC (1591) in the multiple comparisons of the experimental phases. This result indicates that the "monitor with video" phase affected the frequency of resting behaviour differently than the other three phases for the individually housed monkeys. The other behaviours were not affected by the video presentation in either the individually housed or pair-housed monkeys.

\subsection{Habituation to the video presentation}

Individually housed subjects decreased the amount of time they spent watching the video over the progression of observation days, whereas pair-housed subjects watched videos more over time. Fig. 2 shows the frequency of watching behaviour in each day in the "monitor 
1 with video" phase. The GLMM analysis of watching behaviour revealed that the models that

2 included the observation day as the fixed factor (coefficient: -0.021 in the individually housed

3 subjects and 0.023 in the pair-housed subjects) showed significantly lower AICs than the models

4 without it (Table 2). The negative coefficient value in the selected model indicates that the

5 frequency of time spent watching the video decreased with progression of the observation days.

6 In the first five days and last five days, the mean ( \pm SE) number of sampling points with

7 watching behaviour was $22.1( \pm 3.2)$ and $16.9( \pm 2.7)$ for the individually housed subjects and

$8 \quad 5.8( \pm 2.4)$ and $20.8( \pm 4.7)$ for the pair-housed subjects.

9

Fig. 2 about here

the progression of observation days. The models that included the observation day as the fixed

factor (coefficient: -0.025 in the individually housed subjects and -0.011 in the pair-housed subjects) showed significantly lower AICs than the models without it (Fig. 3 and Table 2). The negative coefficient value in the selected model indicates that the frequency of abnormal behaviour decreased with progression of the observation days. In the first five days and last five 
$111.0( \pm 3.1)$ in the individually housed subjects and $8.0( \pm 2.4)$ and $5.1( \pm 2.2)$ in the pair-housed

2 subjects.

3

\subsection{The effect of content of videos}

Both individually housed and pair-housed subjects spent more time watching

"Japanese macaques" videos than other videos (Fig. 4). The models that contained the content

9 as the fixed factor showed significantly lower AICs than the models without the content (Table

10 2). In the multiple comparisons of the contents, the models including "Japanese macaques" at a

11 different level from the other two contents showed the lowest AIC (497.2 in the individually

12 housed subjects and 857.6 in the pair-housed subjects). These results indicate that the content both individually and pair-housed subjects. 
1

Individually housed subjects displayed less abnormal behaviour during videos of conspecifics than the other videos, whereas pair-housed subjects displayed less abnormal behaviour during the videos of people than the other videos (Fig. 5). The models that contained the content as the fixed factor showed significantly lower AICs than the models without the content (Table 2). In the multiple comparisons of the contents, the models including all three contents at different levels showed the lowest AIC (956) in the individually housed subjects and the models including "people" at a different level from the other two contents showed the lowest AIC (786) in the pair-housed subjects. These results indicate that the three different contents affected the frequency of abnormal behaviour differently in the individually housed subjects and that the content "people" affected the frequency of abnormal behaviour differently from the other contents, and there was no difference between the contents "Japanese macaques" and "animation" in the pair-housed subjects.

Fig. 5 about here

\subsection{The effect of controllability over videos}

Controllability did not affect the frequency of abnormal behaviour (Fig. 5). In the individually housed subjects, the model that contained controllability as the fixed factor 
1 (coefficient: 0.019) showed a higher AIC than the model without it (Table 2). In the pair-housed

2 subjects, the model that included controllability as the fixed factor (coefficient: 0.082) showed a

3 lower AIC than the model without it (Table 2). These differences between the models were not

4 significant.

5 Subjects spent more time in the video zone (i.e., the part of the cage that allowed

6 videos to be played) during the days in which they could control the videos than during those in

7 which they had no control. In the analysis of remaining in the video zone (Fig. 6). The models

8 that included controllability as the fixed factor (coefficient: 0.231 in the individually housed

9 subjects and 0.257 in the pair-housed subjects) showed significantly lower AICs than the model

10 that did not include it (Table 2). The positive coefficient values in the selected model indicate

11 that the frequency of staying in the video zone in the controllable days was higher than that in

12 the non-controllable days.

Fig. 6 about here

4. Discussion 
2 Japanese macaques. Both individually and pair-housed subjects displayed fewer abnormal

3 behaviours during video presentation than when the screen was blank. Further, abnormal behaviour in the blank monitor phase was not significantly lower than the control phases, which suggests that it was not the experimental apparatus but the videos that decreased subjects' abnormal behaviour. The video presentation decreased abnormal behaviour of captive Japanese macaques, indicating that it improved their welfare (Young, 2003). pair-housed subjects differently. Although abnormal behaviour of pair-housed monkeys was still reduced by video presentation, the effect of decreasing abnormal behaviour was lower in the

11 individually housed subjects. Resting behaviour decreased during the video presentation only for the individually housed monkeys. These results might be due to several differences, including difference in the baseline frequency of abnormal behaviour and/or activity level, the cage size, and ability to see conspecifics. The individually housed subjects could not see other monkeys during their daily housing condition. The effect of video presentation on individually housed monkeys who can see other conspecifics warrants further investigation.

\subsection{The effect of novelty of videos}


1 video enrichment more effective. In the individually housed subjects, the frequency of watching

2 videos decreased over the duration of the "monitor with video" phase, even though video clips

3 were replaced everyday and were thus novel. This might suggest that the subjects became

4 habituated to the process of watching video enrichment, not to the content of video clips.

5 Therefore, video enrichment should not be used too frequently. Animals often become

6 habituated to frequent presentation of enrichment material (Kuczaj et al., 2002). Nevertheless,

7 the decline of watching behaviour in the present study was less than habituation found in

8 previous studies (Bloomsmith and Lambeth, 2000; Platt and Novak, 1997) in spite of the longer

9 and more frequent presentation than those studies. The frequency of abnormal behaviour of the

10 individually housed subjects also decreased over the duration of the "monitor with video" phase.

11 In the pair-housed subjects, the frequency of watching videos increased and abnormal behaviour

12 decreased as the observation days progressed, suggesting that the habituation to video

13 enrichment in this study was attenuated by the replacement of all video clips everyday. The

14 enrichment procedure in this experiment might be an effective technique to prevent the

15 habituation of animals to videos.

\subsection{The effect of content of videos}

The contents of videos differentially affected the amount of time subjects spent 
1 spent more time watching "Japanese macaques" videos than other videos. They also exhibited

2 fewer abnormal behaviours during this type of video content than other types. As with the individually housed subjects, pair-housed subjects spent more time watching videos of monkeys than other videos. However, they exhibited the fewest abnormal behaviours with videos containing people. These findings indicate that the subjects had a preference for videos of conspecifics, and the contents of videos differentially affect the animals' abnormal behaviour and, therefore, their welfare (Young, 2003). In previous studies, the contents of video enrichment did not affect the behaviour in rhesus macaques (Platt and Novak, 1997) or

9 chimpanzees (Bloomsmith and Lambeth, 2000). The difference of the conclusions between the present study and those studies might be a consequence of the difference of the videos used in

11 the experiments. Various short video clips uploaded on the YouTube website were used as visual enrichment in this study, whereas the previous studies presented 1-h (Platt and Novak, 1997) or 20-min (Bloomsmith and Lambeth, 2000) videos repeatedly in some sessions, so that habituation to the videos might have masked the preference for contents. Animals will watch a preferred video for long time periods. Habituation therefore can occur within the first few sessions, so that the duration of watching it can fall to the same level as that of watching non-preferred videos in later sessions. As a result, the effect of preferred content might have appeared to be equivalent to that of non-preferred content. In the present experiment, the 
1

relatively small habituation resulting from the replacement of the video clips in every session might have resulted in the observed preference for contents for video enrichment.

The subjects in this experiment watched the "Japanese macaques" videos longer than the other videos, suggesting that they had a visual preference for conspecifics. This might appear to conflict with the result of the experiment in Ogura and Matsuzawa (2012). The subjects in Ogura and Matsuzawa (2012) showed clear preferences for videos showing humans and animation, although they could choose to play videos showing conspecifics. The discrepancy between these results might be explained by a difference between the social experiences during the infancy of the subjects. The subjects in the present experiment were mother-reared for at least 1 year after birth, whereas the subjects in Ogura and Matsuzawa (2012) were reared by human caretakers. Developmental history has been suggested to affect visual preference in Japanese macaques (Fujita, 1990, 1993), gibbons (Tanaka and Uchikoshi, 2010), chimpanzees (Tanaka, 2003, 2007), and gorillas (Maloney et al., 2011). To enhance the effect of video enrichment, the contents of videos could be changed based on animals' developmental history or individual differences.

\subsection{The effect of controllability over videos}

The subjects stayed in the video zone longer in the controllable days than in the non-controllable days, suggesting that they preferentially chose video presentation. The IRD 
1 system employed in the present study was helpful to satisfy the monkeys' preference for videos

2 and control over videos. However, even though subjects changed their location in the cages

3 depending on whether or not they had control over the videos, this control did not clearly affect

4 the level of abnormal behaviour in the present experiment.

5

The procedure which enabled the monkeys to control videos might have a potential for

6 improvement. The pair-housed monkeys watched the videos less when they had control than

7 when they did not. They also stayed at video zone for shorter periods of time than individually

8 housed subjects due to their larger cage size. Therefore, playing duration of videos when the

9 monkeys had control was much shorter than they did not. This explains the shorter watching

10 duration when the pair-housed monkeys had control over video presentation. My system

11 provided control of videos to only one animal. How to provide control to multiple animals

12 living together needs further consideration.

\subsection{General discussion}

The present study demonstrated that the captive Japanese macaques watched video

presentations, which decreased abnormal behaviour. Reduction of abnormal behaviour is

considered to be evidence that environmental enrichment improves animal welfare (Young,

17 2003). The results here showed that video presentation can improve the welfare of captive 
Environmental enrichment must be implemented within the limitations of time, human,

2

and financial resources, as well as species-specific characteristics of the subjects (Kreger et al., 1998). Two newly developed technologies, namely, a program of automated presentation of network-shared videos and an IRD sensor, were incorporated into the video presentation device used in the present experiment. Both of these technologies helped save financial and human costs of environmental enrichment. The computer program automatically presented novel and preferred videos by accessing a video-sharing website (YouTube), which was less costly and time intensive than having caretakers prepare or purchase videos, something done at many facilities. Utilization of an IRD sensor also reduced the time needed for the training required to provide animals with control over the environment. By employing the IRD sensor, which allowed detection of the distance to an animal, it was possible to make the video presentation contingent on the monkey's location in the cage. This, in turn, allowed the monkeys to control the videos with natural behaviour, and without any specific training by humans. The presentation device tested in the present experiment can contribute to the welfare of the monkeys with low human cost.

The findings of the present study provide some valuable suggestions for general implementation of environmental enrichment. In the present study, the video enrichment was implemented based on an understanding of the cognitive abilities of Japanese macaques. They 
1 have preferences for the novelty and content of videos (Ogura and Matsuzawa, 2012). Having

2 control over the visual environment is valuable for captive Japanese macaques (Ogura, 2011).

3 These cognitive abilities are classified into the input and output process, respectively, according

4 to the perspective of cognitive enrichment (Morimura, 2006). In the present experiment, the

5 stimulation of such process affected monkeys' behaviour. This study has provided some

6 empirical evidence that, by taking such properties as input and output processes into

7 consideration, environmental enrichment makes the captive environment more stimulating and

8 supplies captive animals with opportunities to exert their ability to cope with the environment.

9 Implementation of environmental enrichment based on the species-specific characteristics can

10 improve animal welfare. Therefore, understanding the species-specific characteristics of subject

11 animals is essential for appropriate implementation of environmental enrichment.

\section{Conclusion}

Abnormal behaviour of captive Japanese macaques could be ameliorated by exposure to video presentation. This was achieved at low cost by using developed technologies such as network-shared videos and an IRD sensor, which maintained the novelty of videos, provided

17 preferred video content, and allowed subjects to have control over their visual world.

18 Implementation of video enrichment based on an understanding of species-specific cognitive 
1 characteristics of Japanese macaques increased their welfare. Overall, the present study provides empirical evidence that video enrichment has positive effects on the welfare of captive animals and can be a possible environmental enrichment for them. of Education, Culture, Sports, Science and Technology grant \#16002001 and \#20002001 to

9 Tetsuro Matsuzawa, and \#19300091 to Masaki Tomonaga, and Japan Society for the Promotion of Science-gCOE Programs A06 and D07 of Kyoto University and HOPE project of the Primate members of the Center for Human Evolution Modeling Research, Primate Research Institute, Kyoto University, for their management of the subjects' health. Special thanks are due to Dr. E. Nakajima, the Graduate School of Science, Kyoto University and Dr. Kristine Coleman, the

17 is a part of the author's $\mathrm{Ph}$. D. thesis. This work complied with the laws of Japan, and housing 
1 Primates produced by the Primate Research Institute, Kyoto University (2nd ed., 2002). 
2 Akaike, H., 1974. A new look at the statistical model identification. IEEE Trans. Automat. Contr.

Altmann, J., 1974. Observational study of behavior: Sampling methods. Behav. Brain. Res. 49,

Andrews, M.W., Rosenblum, L.A., 2001. Effects of change in social content of video rewards on response patterns of bonnet macaques. Learn. Motiv. 32, 401-408.

Bloomsmith, M.A., Brent, L.Y., Schapiro, S.J., 1991. Guidelines for developing and managing an environmental enrichment program for nonhuman primates. Lab. Anim. Sci. 41, 327-377.

Bloomsmith, M.A., Lambeth, S.P., 2000. Videotapes as enrichment for captive chimpanzees (Pan troglodytes). Zoo Biol. 19, 541-551.

Brannon, E.M., Andrews, M.W., Rosenblum, L.A., 2004. Effectiveness of video of conspecifics as a reward for socially housed bonnet macaques (Macaca radiata). Percept. Mot. Skills. 98, 849-858.

Brent, L., Stone, A.M., 1996. Long-term use of televisions, balls, and mirrors as enrichment for paired and singly caged chimpanzees. Am. J. Primatol. 39, 139-145. 

technology to enhance zoological parks. Zoo Biol. 30, 487-497.

2 Dobson, A.J., 2002. An introduction to generalized linear models - Second edition. Chapman \& Hall, London.

Fujita, K., 1990. Species preference by infant macaques with controlled social experience. Int. J.

Fujita, K., 1993. Development of visual preference for closely related species by infant and juvenile macaques with restricted social experience. Primates. 34, 141-150.

Hanson, J.D., Larson, M.E., Snowdon, C.T., 1976. The effects of control over high intensity noise on plasma cortisol levels in rhesus monkeys. Behav. Biol. 16, 333-340.

Inglis, I.R., Forkman, B., Lazarus, J., 1997. Free food or earned food? A review and fuzzy model of contrafreeloading. Anim. Behav. 53, 1171-1191.

Koba, R., Izumi, A., 2008. Japanese monkeys (Macaca fuscata) discriminate between pictures of conspecific males and females without specific training. Behav. Processes. 79, 70-73.

Kreger, M.D., Hutchins, M., Fascione, N., 1998. Context, ethics, and environmental enrichment in zoos and aquariums. In: Shepherdson, D.J., Mellen, J.D., Hutchins, M. (Eds.), Second nature: Environmental enrichment for captive animals. Smithsonian 
1 Kuczaj, S., Lacinak, T., Fad, O., Trone, M., Solangi, M., Ramos, J., 2002. Keeping environmental enrichment enriching. Int. J. Comp. Psychol. 15, 127-137.

Lee, G.H., Yi, M.J., Crockett, C.M., 2011. Assessing video presentation as enrichment for captive male pigtailed macaques (Macaca nemestrina). Lab. Primate Newsl. 50, 7-9.

Mallapur, A., Choudhury, B. C., 2003. Behavioral abnormalities in captive nonhuman primates. J. Appl. Anim. Welf. Sci. 6, 275-284.

Maloney, M.A., Leighty, K.A., Kuhar, C.W., Bettinger, T.L., 2011. Behavioral responses of silverback gorillas (Gorilla gorilla gorilla) to videos. J. Appl. Anim. Welf. Sci. 14, 96-108.

Menzel, E.W., 1991. Chimpanzees (Pan troglodytes) - Problem seeking versus the bird-in-hand, least-effort strategy. Primates. 32, 497-508.

Morimura, N., 2006. Cognitive enrichment in chimpanzees: An approach of welfare entailing an animal's entire resources. In: Matsuzawa, T., Tomonaga, M., Tanaka, M. (Eds.), Cognitive development in chimpanzees. Springer-Verlag, Tokyo, pp 368-391.

Murai, C., Tomonaga, M., Kamegai, K., Terazawa, N., Yamaguchi, M.K., 2004. Do infant Japanese macaques (Macaca fuscata) categorize objects without specific training? Primates. 45, 1-6. 
1 Ogura, T., 2011. Contrafreeloading and the value of control over visual stimuli in Japanese

macaques (Macaca fuscata). Anim. Cogn. 14, 427-431.

Ogura, T., Matsuzawa, T., 2012. Video preference assessment and behavioral management of single-caged Japanese macaques (Macaca fuscata) by movie presentation. J. Appl. Anim. Welf. Sci. 15, 101-112.

Platt, D.M., Novak, M.A., 1997. Videostimulation as enrichment for captive rhesus monkeys (Macaca mulatta). Appl. Anim. Behav. Sci. 52, 139-155.

Quinn, P.C., Yahr, J., Kuhn, A., Slater, A.M., Pascalis, O., 2002. Representation of the gender of human faces by infants: A preference for female. Perception. 31, 1109-1121.

Reinhardt, V., 1994. Caged rhesus macaques voluntarily work for ordinary food. Primates. 35, 95-98.

Reinhardt, V. (Ed.), 2010. Caring hands: Discussion by the laboratory animal refinement \& enrichment forum volume 2. Animal Welfare Institute, Washington, DC.

Sambrook, T.D., Buchanan-Smith, H.M., 1997. Control and complexity in novel object enrichment. Anim. Welf. 6, 207-216.

Shepherdson, D.J., 1998. Introduction: Tracing the path of environmental enrichment in zoos. In: Shepherdson, D.J., Mellen, J.D., Hutchins, M. (Eds.), Second nature: Environmental enrichment for captive animals. Smithsonian Institution Press, 
1

2 Tanaka, M., 2003. Visual preference by chimpanzees (Pan troglodytes) for photos of primates

9 Walsh, S., Bramblett, C.A., Alford, P.L., 1982. A vocabulary of abnormal behaviors in 
2 Fig. 1. Mean ( \pm SE) frequency of abnormal behaviour in each experimental phase. In the first and second no-monitor phases, the enrichment device was not present. In the blank monitor phase, the apparatus was present, but the display showed no videos. In the "monitor with video"

5 phase, the subjects could watch videos on the display. SEs are based on the error across all scores from all subjects.

8 Fig. 2. The frequency of watching behaviour in each day in the "monitor with video" phase. (a)

9 individually housed subjects, (b) pair-housed subjects.

11 Fig. 3. The frequency of abnormal behaviour in each day in the "monitor with video" phase. (a)

12 individually housed subjects, (b) pair-housed subjects.

14 Fig. 4. Mean ( \pm SE) frequency of watching behaviour in each presentation condition in the "monitor with video" phase. The "monkey", "people", and "animation" indicate the content of the video clips. In the days of control "+", the subjects could watch the videos only when they

17 stayed in the video zone in the cage. In the days of control "-", the videos were shown on the 

pair-housed subjects.

Fig. 5. Mean $( \pm \mathrm{SE})$ frequency of abnormal behaviour in each presentation condition in the "monitor with video" phase. The "monkey", "people", and "animation" indicate the content of

6 the video clips. In the days of control "+", the subjects could watch the videos only when they

7 stayed in the video zone in the cage. In the days of control "-", the videos were shown on the

8 display continuously irrespective of the monkey's position in the cage. The order of the conditions was randomized among the subjects. (a) individually housed subjects, (b) pair-housed subjects.

Fig. 6. Mean ( \pm SE) frequency of staying in the video zone in each presentation condition in the "monitor with video" phase. The "monkey", "people", and "animation" indicate the content of the video clips. In the days of control "+", the subjects could watch the videos only when they stayed in the video zone in the cage. In the days of control "-", the videos were shown on the display continuously irrespective of the monkey's position in the cage. The order of the 
Fig. 1

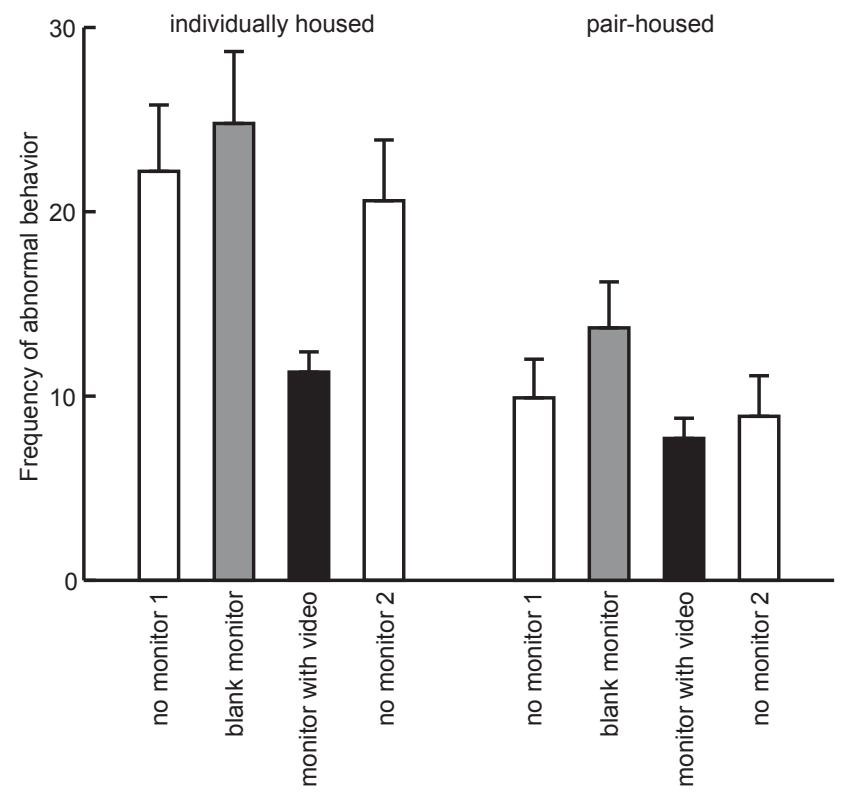


Fig. 2

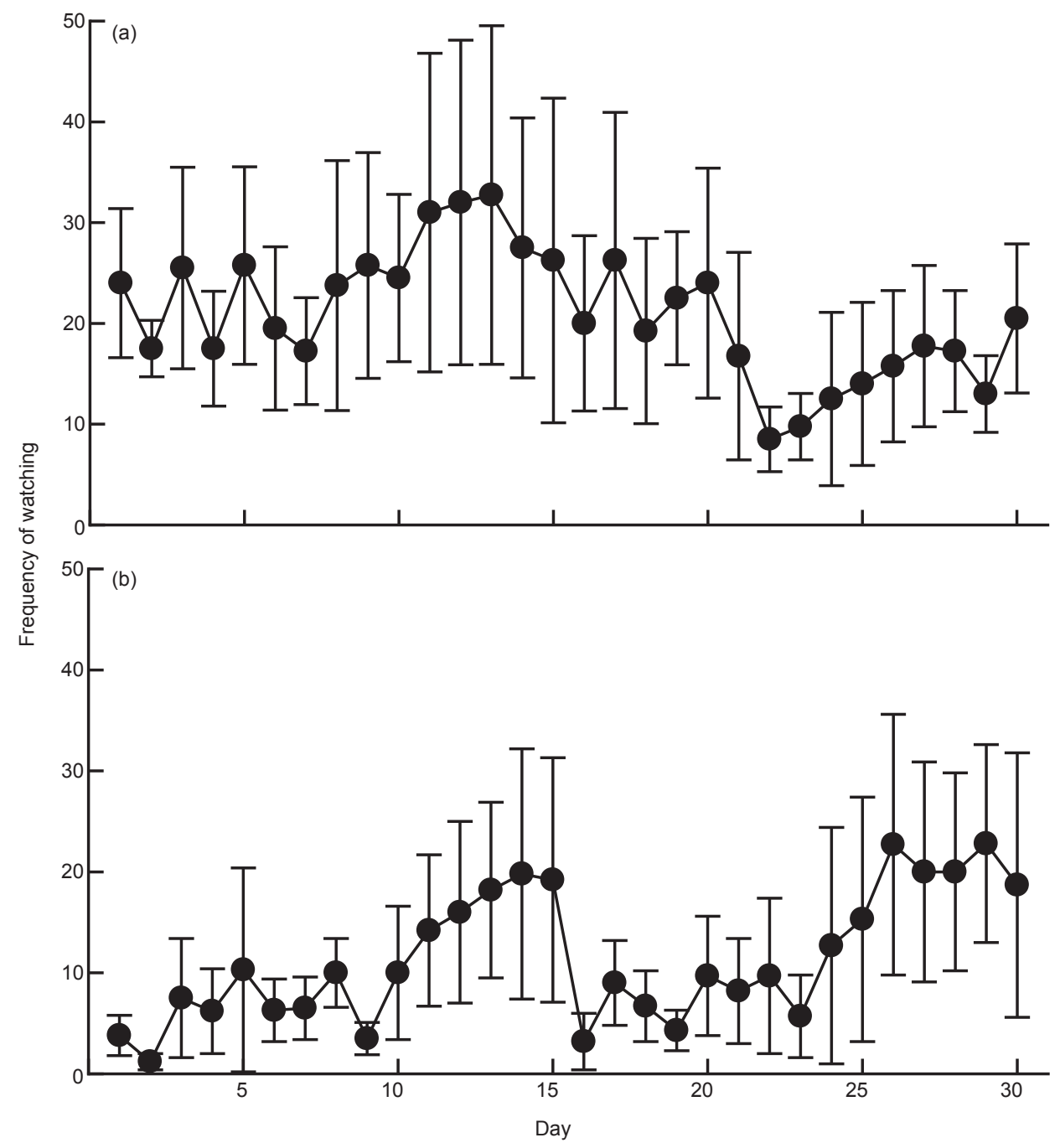


Fig. 3

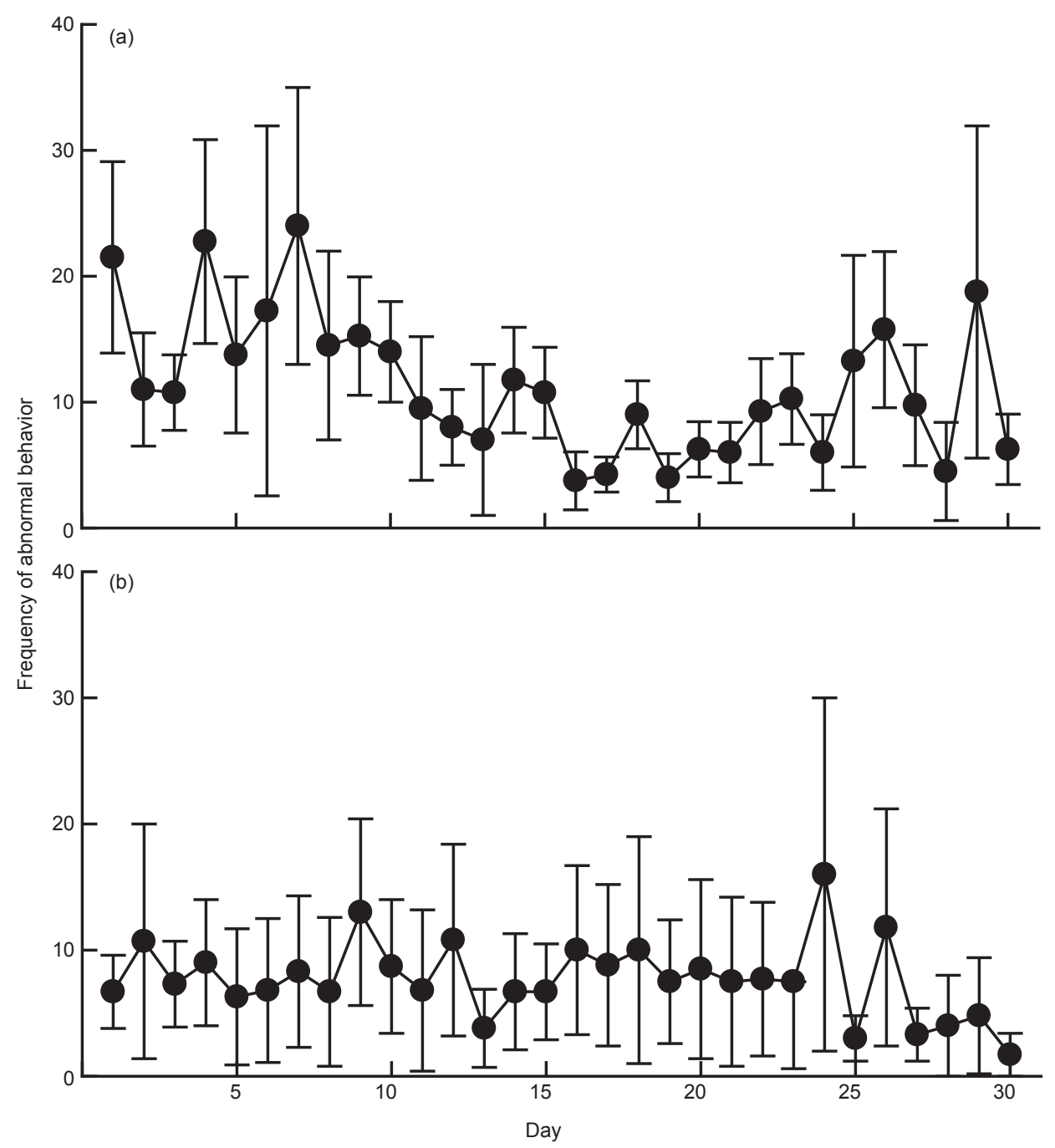


Fig. 4

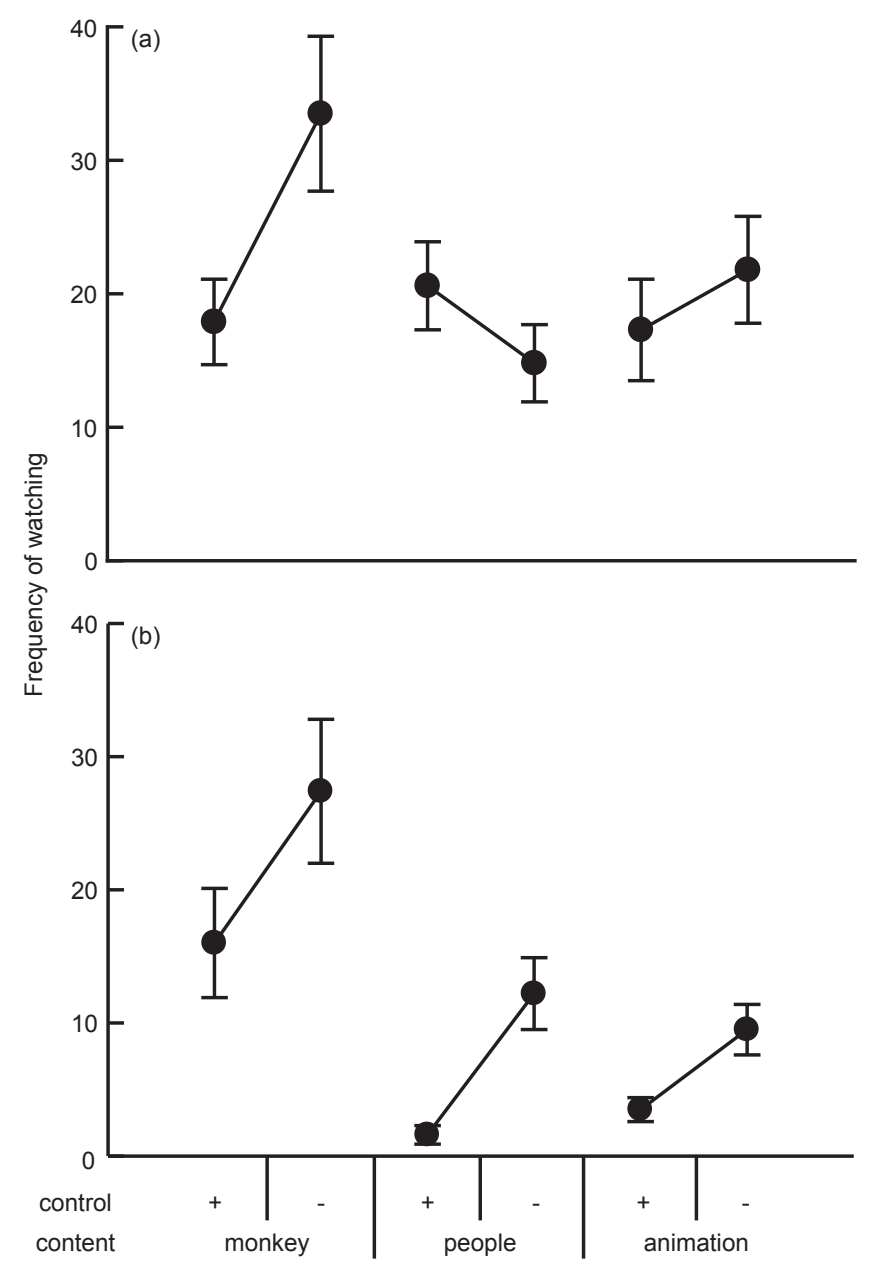


Fig. 5

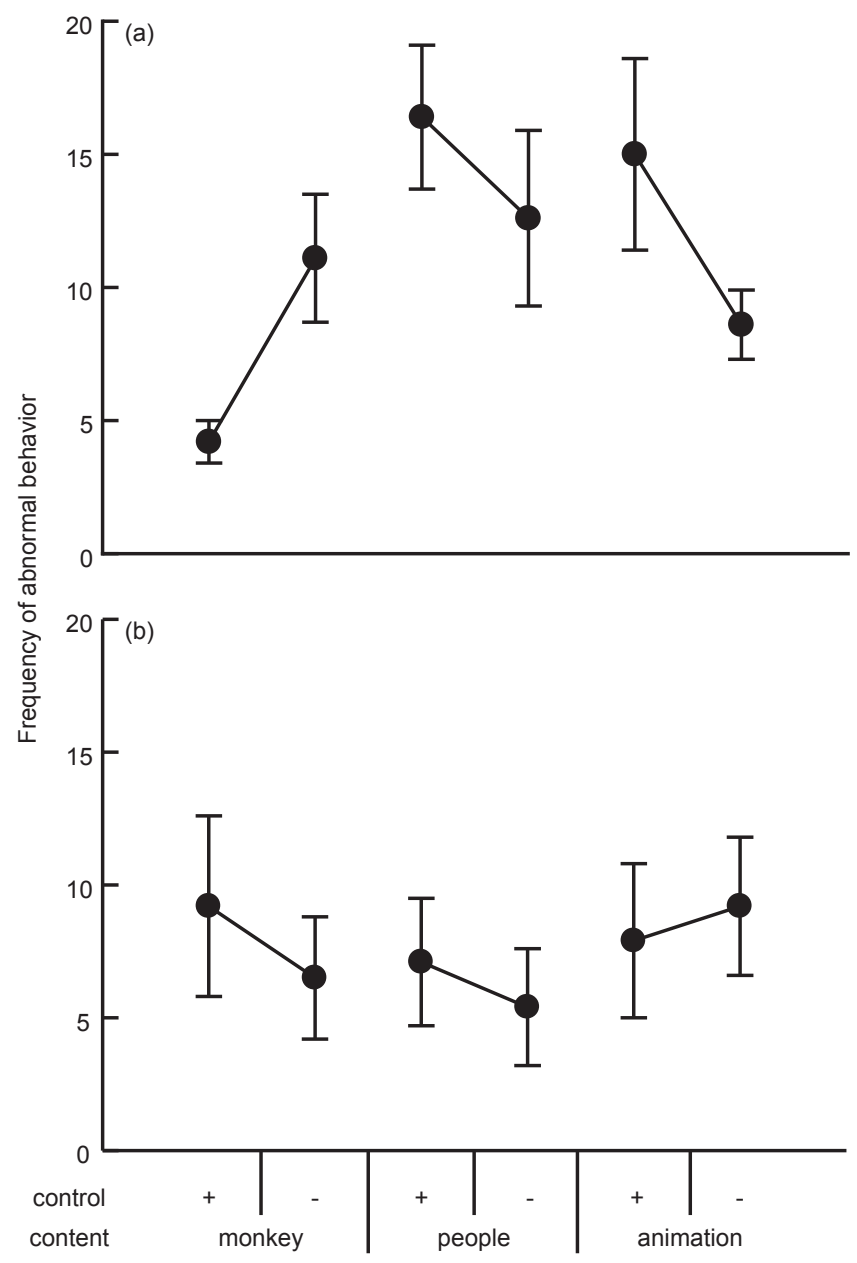


Fig. 6

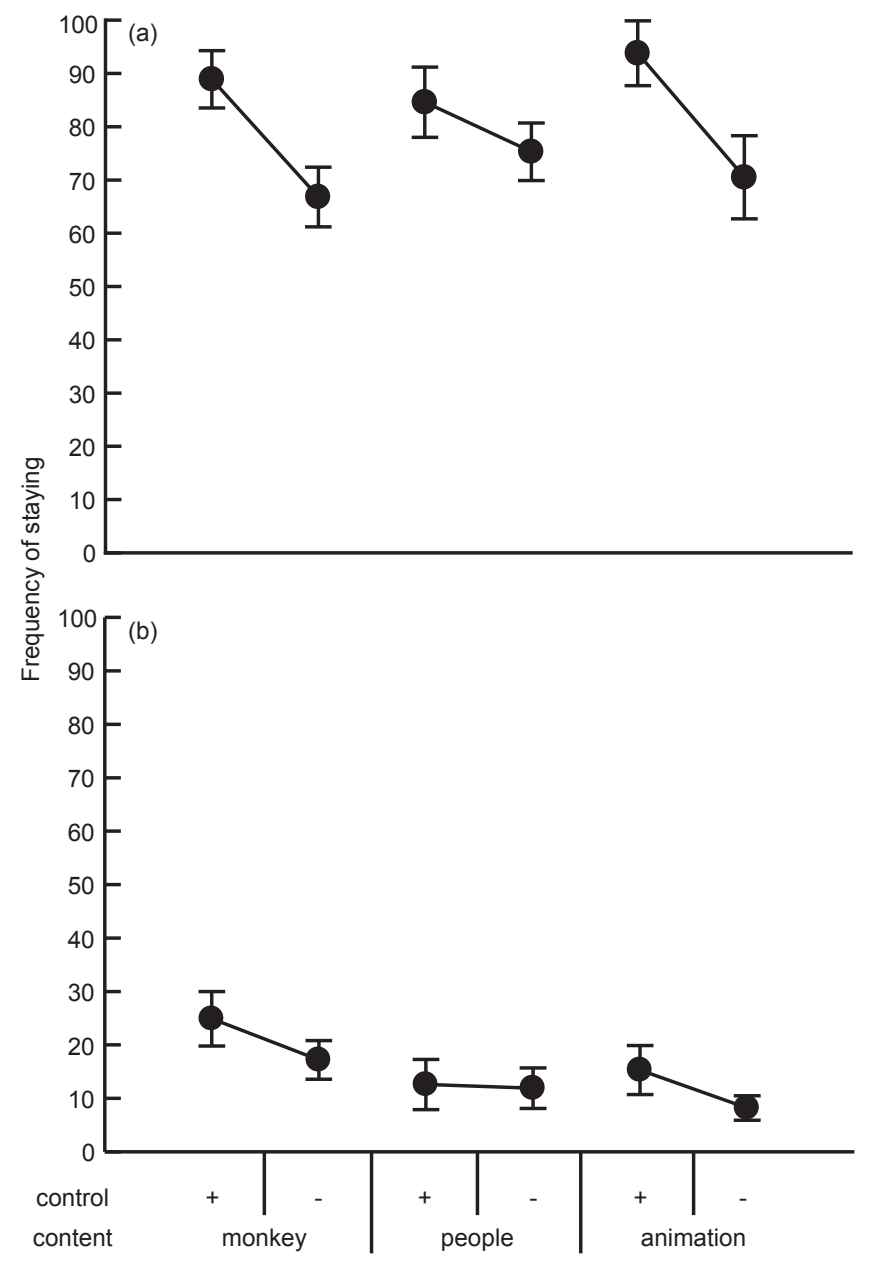




\section{Table 1}

List of behaviours used for behavioural observation in the present study.

\section{Abnormal behaviour}

Autoerotic stimulation Self-directed sexual activity and masturbation

Floating limb Unusual movement pattern of a limb; after visually tracking for a short time, the floating limb is often attacked viciously by oneself

Self-clasping Use of hands or feet to hold onto part of body by oneself

Self-biting Hands, legs, arms and/or torso bitten in a stereotyped fashion by oneself

Stereotypic pacing

Rocking

Saluting/eye poking

Rubbing bars

Licking bars

Licking hand

Hair plucking
Repetitive pacing along the same path

Repetitive seated, bipedal, or quadrupedal rocking

\begin{tabular}{cl}
\hline Rubbing bars & Repetitive rubbing bars of cages with hands \\
\hline Licking bars & Repetitive licking of bars \\
\hline Licking hand & Hands or foot licked by oneself \\
\hline Hair plucking & Pulling out of own or another animal's hair \\
\hline Feeding & Reaching hand into a food box and putting food into mouth \\
\hline
\end{tabular}


Locomotion

Moving bipedally or quadrupedally

Self-grooming

Pushing own hair aside and picking up something

Social behaviour

Allogrooming, mounting, and agonistic and submissive behaviour

Watching video

Directing head and eyes to the display during video presentation

Resting

Staying still without any other behaviour 


\title{
Table 2
}

Results of Generalized Linear Mixed Model analyses of behavioural observation.

Individually housed subjects Pair-housed subjects

Likelihood ratio test

$\mathrm{AIC}^{\mathrm{a}}$

none $e^{\mathrm{c}}$

3473

1979

Habituation to the video presentation

observation day, content, and

Watching the video

498.

71.216

$<0.001$

859.5

$66.65<0.001$

controllability

content and controllability

567.8

924.1

observation day, content, and

\begin{abstract}
Abnormal behaviour
\end{abstract}
controllability

content and controllability

1017

798

The effect of content of videos

Watching the video

observation day, content, and

498.6

70.552

$<0.001 \quad 859.5$

$601.5<0.001$ 
controllability

observation day and controllability $\quad 565.1$

observation day, content, and

Abnormal behaviour

956.

81.649

$<0.001$

787.5

$18.79<0.001$

controllability

observation day and controllability

1034

802.3

The effect of controllability over videos

observation day, content, and

\begin{tabular}{|c|c|c|c|c|c|c|c|}
\hline \multirow[t]{2}{*}{ Abnormal behaviour } & \multicolumn{6}{|l|}{ controllability } & 0.13 \\
\hline & observation day and content & 954.2 & & & 787.8 & & \\
\hline & observation day, content, and & & & & & & \\
\hline \multirow[t]{3}{*}{ Location in the cage } & & 943.9 & 126.9 & $<0.001$ & 3141 & 40.59 & $<0.001$ \\
\hline & \multicolumn{7}{|l|}{ controllability } \\
\hline & observation day and content & 1069 & & & 3180 & & \\
\hline
\end{tabular}

a AIC means the "Akaike Information Criterion", the index used to compare the fitted models.

The model with the lower AIC is preferred.

${ }^{\mathrm{b}}$ The experimental phase indicates the four conditions, under which observation was conducted,

such as no-monitor 1, blank monitor, monitor with video, and no-monitor 2.

${ }^{\mathrm{c}}$ This model contained no fixed factor. 